\title{
Prevalence of asymptomatic bronchiectasis and associations among the health screening population in South Korea
}

\author{
Soo Han Kim¹, Young Ju Jung ${ }^{2,3}$, Myung-Su Ko ${ }^{3}$, Sei Won Lee ${ }^{2}$, Jae Seung Lee ${ }^{2}$ and Yeon-Mok $\mathrm{Oh}^{2}$
}

${ }^{1}$ Dept of Internal Medicine, Biomedical Research Institute, Pusan National University Hospital, Seo-gu, Busan, Korea. ${ }^{2}$ Dept of Pulmonary and Critical Care Medicine, University of Ulsan College of Medicine, Asan Medical Centre, Songpa-gu, Seoul, Korea. ${ }^{3} \mathrm{Health}$ Screening and Promotion Centre, Asan Medical Centre, Songpa-gu, Seoul, Korea.

Corresponding author: Yeon-Mok Oh (ymoh55@amc.seoul.kr)

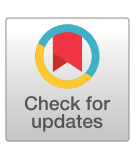

Copyright @The authors 2021
This version is distributed under
the terms of the Creative
Commons Attribution Non-
Commercial Licence 4.0. For
commercial reproduction rights
and permissions contact
permissions@ersnet.org
This article has supplementary
material available from
openres.ersjournals.com.
Received: 6 April 2021
Accepted: 25 May 2021

Shareable abstract (@ERSpublications)

The prevalence of asymptomatic bronchiectasis appears to be more common than that of symptomatic bronchiectasis. Female sex, older age, liver disease, COPD, history of tuberculosis and low $\mathrm{FEV}_{1}$ may be the factors related to asymptomatic bronchiectasis. https://bit.ly/3bVSodN

Cite this article as: Kim SH, Jung YJ, Ko M-S, et al. Prevalence of asymptomatic bronchiectasis and associations among the health screening population in South Korea. ERJ Open Res 2021; 7: 00188-2021 [DOI: 10.1183/23120541.00188-2021].

\section{Abstract}

Despite the burden of bronchiectasis, there is little data from South Korea regarding asymptomatic bronchiectasis. We aimed to investigate the prevalence of bronchiectasis based on computed tomography (CT) findings, with emphasis on asymptomatic bronchiectasis (CT-proven) and its related factors.

We analysed data of individuals who underwent chest CT at a South Korean health screening centre from 2016 to 2017. The prevalence of bronchiectasis was evaluated and adjusted by sex, age, and corresponding year for the Korean general population. Logistic regression analysis was performed to identify factors related to asymptomatic bronchiectasis by comparison between a group without bronchiectasis and a group with symptomatic bronchiectasis.

Among the 27617 subjects screened, 1005 were diagnosed with bronchiectasis based on CT findings, representing an adjusted prevalence of 2329 out of 100000 . The adjusted prevalence of asymptomatic bronchiectasis was 1235 out of 100000 , and that of symptomatic bronchiectasis was 1094 out of 100000 . Compared with the non-bronchiectasis group, the factors related to asymptomatic bronchiectasis were female sex (OR 1.41; 95\% CI 1.18-1.70), older age (OR 1.06; 95\% CI 1.05-1.07), comorbid liver disease (OR 1.32; 95\% CI 1.07-1.63) or COPD (OR 4.99; 95\% CI 2.88-8.64), history of tuberculosis (OR 1.98; 95\% CI 1.46-2.68) and low forced expiratory volume in 1 s (FEV 1 ; OR 0.99; 95\% CI 0.98-0.998).

In South Korea, the prevalence of asymptomatic bronchiectasis appeared higher than that of symptomatic bronchiectasis. Female sex, older age, liver disease, COPD, history of tuberculosis and low $\mathrm{FEV}_{1}$ may be the factors related to asymptomatic bronchiectasis.

\section{Introduction}

Bronchiectasis is a chronic respiratory disease characterised by irreversible dilatation of the bronchus and/ or its branches, along with recurrent airway inflammation and infection [1]. It is suggested that bronchiectasis may develop by a vicious cycle of impaired mucus clearance, chronic infection, airway obstruction, inflammation and progressive tissue destruction [2]. For diagnosis of bronchiectasis, chest computed tomography (CT) is now used as a reference standard [3, 4].

Although bronchiectasis might be clinically important as a chronic respiratory disease with irreversible changes, it was once considered as an orphan disease and often neglected [5]. This might be attributed to lack of a standard diagnostic tool and treatment strategy, along with a disinterest and misperception that bronchiectasis was uncommon [6]. Recently, a longitudinal study in the UK focusing on older age groups showed an increase in the annual incidence and prevalence of bronchiectasis from 2004 to 2013 [7]. Another study in the United States showed an increase in bronchiectasis prevalence $(8.7 \%$ per year from 2000 to 2007) [8]. Accordingly, bronchiectasis has imposed a considerable burden on public health, 
including mortality, hospital admission and high costs of healthcare resources $[8,9]$. However, previous epidemiological information has been limited to retrospective, cross-sectional studies, with the data mainly extracted using the International Classification of Diseases 10th revision (ICD-10) diagnostic code and mostly based on hospital discharge, medical insurance or partially ad hoc population-based databases [8, 10 , 11]. Thus, real-world data on the epidemiology of bronchiectasis is still limited for most countries, including South Korea.

Bronchiectasis is also heterogeneous, showing a wide range of clinical features [12]. Some patients may be asymptomatic, whereas others may have chronic productive cough, dyspnoea, recurrent chest infections and occasional haemoptysis [13]. The presence of symptoms has clinically important implications. Combined with microbiology, symptoms are used in classifying bronchiectasis phenotypes, with each phenotype having distinctive features regarding quality of life, exacerbations, hospitalisations and mortality [14]. However, studies on the characteristics of bronchiectasis with clinical symptoms are limited.

Recent studies have shown some advances in the treatment of bronchiectasis, leading to better prognosis [15]. Therefore, it is important to identify and treat bronchiectasis in its early stages, starting from the asymptomatic period. However, there are limited data regarding the prevalence of asymptomatic bronchiectasis and its related factors.

Therefore, we aimed to evaluate the real-world prevalence of bronchiectasis based on chest CT findings at a health screening centre of Asan Medical Centre in South Korea. Furthermore, we aimed to investigate the prevalence and factors associated with asymptomatic bronchiectasis (CT-proven). To identify these related factors, we analysed the group of subjects with asymptomatic bronchiectasis and compared them to the groups without bronchiectasis and with symptomatic bronchiectasis.

\section{Materials and methods}

\section{Subjects}

This cross-sectional, retrospective study was performed using the database from the in-house electronic medical record system at the Health Screening and Promotion Centre of Asan Medical Centre, Seoul, South Korea, from 2016 to 2017. We extracted the data of 27641 subjects aged $\geqslant 18$ years who underwent chest CT during their routine medical check-ups. CT scans were performed using a multidetector-raw CT (MDCT) scanner.

\section{Identification of bronchiectasis and patients with asymptomatic bronchiectasis}

Bronchiectasis was identified by radiologists using chest CT findings of broncho-arterial ratio $>1$, lack of tapering, airway visibility within $1 \mathrm{~cm}$ of the costopleural surface or touching the mediastinal pleura [4]. Symptoms of bronchiectasis were defined as the presence of at least one of the following five symptoms: cough, sputum production, dyspnoea, wheezing and haemoptysis [2]. Asymptomatic bronchiectasis was defined as the presence of bronchiectatic findings on chest CT with none of the above-mentioned symptoms. Contrarily, symptomatic bronchiectasis was defined as the presence of bronchiectatic findings on chest CT with any of the above-mentioned five symptoms. As a control group, non-bronchiectasis was defined as the absence of bronchiectatic findings on chest CT and the absence of any of the five respiratory symptoms. We compared the group with asymptomatic bronchiectasis to those with non-bronchiectasis and symptomatic bronchiectasis. Symptomatic patients without bronchiectasis were excluded for comparison.

\section{Data for related factors}

Information on age, sex, height, weight, comorbidities (malignancy, hypertension, diabetes mellitus, hyperlipidaemia, angina, heart failure, arrhythmia, peripheral vascular disease, cerebrovascular disease (CVD), osteoporosis, arthritis, dementia, liver disease, kidney disease, COPD, asthma, tuberculosis, gastro-oesophageal reflux disease (GERD), inflammatory bowel disease, allergic rhinitis), smoking history, and respiratory symptoms at enrolment (dyspnoea, cough (persisting for at least 2 months), sputum production (persisting for at least 3 months per year in the past 2 years), wheezing, haemoptysis) was obtained using standard self-reporting questionnaires developed at the health screening centre during the first visit or CT scan [16]. Body mass index (BMI) was calculated as weight in kilograms divided by the square of height in metres $\left(\mathrm{kg} \cdot \mathrm{m}^{-2}\right)$. According to the National Institutes of Health (NIH), BMI was stratified into three groups: underweight (BMI $<18.5 \mathrm{~kg} \cdot \mathrm{m}^{-2}$ ), normal weight (BMI $\geqslant 18.5$ and $<25 \mathrm{~kg} \cdot \mathrm{m}^{-2}$ ), overweight or obese (BMI $\geqslant 25 \mathrm{~kg} \cdot \mathrm{m}^{-2}$ ) [17]. Spirometric data, including forced expiratory volume in $1 \mathrm{~s}\left(\mathrm{FEV}_{1}\right)$, forced vital capacity (FVC) and $\mathrm{FEV}_{1} / \mathrm{FVC}$ were measured according to the American Thoracic Society/European Respiratory Society Guidelines [18]. The results were expressed as percentages of the normal predicted values. Blood haemoglobin $\left(\mathrm{g} \cdot \mathrm{dL}^{-1}\right)$ and C-reactive protein $(\mathrm{CRP})$ levels $\left(\mathrm{mg} \cdot \mathrm{dL}^{-1}\right)$ were also obtained during stable state. 


\section{Prevalence adjustment}

The crude prevalence of bronchiectasis was calculated as the number of subjects with bronchiectasis divided by the total number of subjects who underwent chest CT for health screening. To adjust for unequal probabilities of selection and to account for non-participation, the adjusted prevalence was calculated based on the sampling weight. Results were post-stratified to the Korean population as estimated by the Bureau of the Census 2016-2017, with further stratification by either sex or age [19].

\section{Statistical analyses}

Continuous data are presented as mean \pm SD or median (25th-75th interquartile range). Categorical data are presented as absolute numbers with percentages in parentheses. Data among the three groups were compared using the analysis of variance t-test or Kruskal-Wallis test for continuous data and the Chi-square or linear-by-linear test for categorical data. Data between two groups were compared using the t-test or Mann-Whitney test for continuous data and the Chi-square or Fisher's exact test for categorical data. Univariate and multivariate logistic regression analyses were performed to identify risk factors for asymptomatic bronchiectasis. Univariate analyses were performed for all baseline characteristic variables. Multivariate analyses were performed with age, sex and variables whose p-values were $<0.10$ in univariate analyses. Odds ratios (95\% confidence intervals) were computed for each variable. Statistical analyses were performed using IBM SPSS Statistics Version 24 (IBM Corp., Armonk, NY, USA). A p-value <0.05 was considered statistically significant.

\section{Institutional review board approval and informed consent}

This study was approved by the Institutional Review Board (IRB) of Asan Medical Centre (IRB No. 2018-0691). The requirement for informed consent from the subjects was waived because of the retrospective study design.

\section{Results}

\section{Study population}

A total of 27617 subjects who underwent chest CT from 2016 to 2017 were included in our study after excluding 24 subjects with missing data (figure 1). Their mean age was $58.4 \pm 8.9$ years, and the proportion of males was greater than females ( $\mathrm{n}=17511 ; 63.4 \%)$. Among the 27617 subjects, 1005 were diagnosed with bronchiectasis based on CT results. Among the 1005 subjects with bronchiectasis, the mean age was $62.4 \pm 7.5$ years, and the proportion of males was greater than females $(n=585 ; 58.2 \%)$. The numbers of subjects were 16143, 566 and 439 in the non-bronchiectasis group, the asymptomatic bronchiectasis group and the symptomatic bronchiectasis group, respectively.

\section{Baseline characteristics}

The baseline characteristics of the three groups are shown in table 1, figure 2 and supplementary table S1. The proportions of females were different among the three groups, with an increasing trend towards the symptomatic bronchiectasis group ( $34.4 \%$ versus $39.0 \%$ versus $45.3 \%, \mathrm{p}<0.001$ ), whereas age was greater in the asymptomatic bronchiectasis group than in the other two groups (58.19 years versus 64.24 years versus 62.64 years, $\mathrm{p}<0.001$ ). Comorbidities, such as hypertension, hyperlipidaemia, arrhythmia, osteoporosis, arthritis, dementia, liver disease, kidney disease, COPD, asthma, tuberculosis, GERD and allergic rhinitis, were also different among the three groups. Furthermore, smoking history (53.2\% versus $46.5 \%$ versus $44.6 \%, \mathrm{p}<0.001)$ and $\mathrm{FEV}_{1}(92.06 \%$ of the predicted value versus $90.42 \%$ versus $85.63 \%, \mathrm{p}<0.001$ ) were different, with decreasing trends towards the symptomatic bronchiectasis group. Regarding laboratory findings, haemoglobin levels were different, with a decreasing trend towards the symptomatic bronchiectasis group $\left(14.61 \mathrm{~g} \cdot \mathrm{dL}^{-1}\right.$ versus $14.44 \mathrm{~g} \cdot \mathrm{dL}^{-1}$ versus $14.38 \mathrm{~g} \cdot \mathrm{dL}^{-1}, \mathrm{p}<0.001$ ), whereas CRP levels were relatively higher in the symptomatic bronchiectasis group than in the other groups $\left(0.11 \mathrm{mg} \cdot \mathrm{dL}^{-1}\right.$ versus $0.11 \mathrm{mg} \cdot \mathrm{dL}^{-1}$ versus $\left.0.16 \mathrm{mg} \cdot \mathrm{dL}^{-1}, \mathrm{p}<0.001\right)$.

\section{Prevalence of bronchiectasis}

The crude prevalence of bronchiectasis was 3639 per 100000 subjects, with a higher prevalence among females than males (4156 per 100000 versus 3341 per $100000 ; \mathrm{p}<0.001$ ). The prevalence of bronchiectasis, adjusted by age, sex and year, was 2329 per 100000 . The crude prevalence of bronchiectasis increased substantially with age (from 0 per 100000 in ages 20-29 years to 7210 per 100000 in ages $\geqslant 70$ years; $\mathrm{p}<0.001$ ) (figure 3 ). Across all age ranges with 10 -year intervals, the prevalence of bronchiectasis was higher in females than in males, especially within the range of 50-59 years (3641 per 100000 versus 2409 per 100000 ; $\mathrm{p}<0.001$ ).

Based on the presence of respiratory symptoms, there were 566 cases of asymptomatic bronchiectasis and 439 cases of symptomatic bronchiectasis among the total 27617 subjects. The crude prevalence in the 


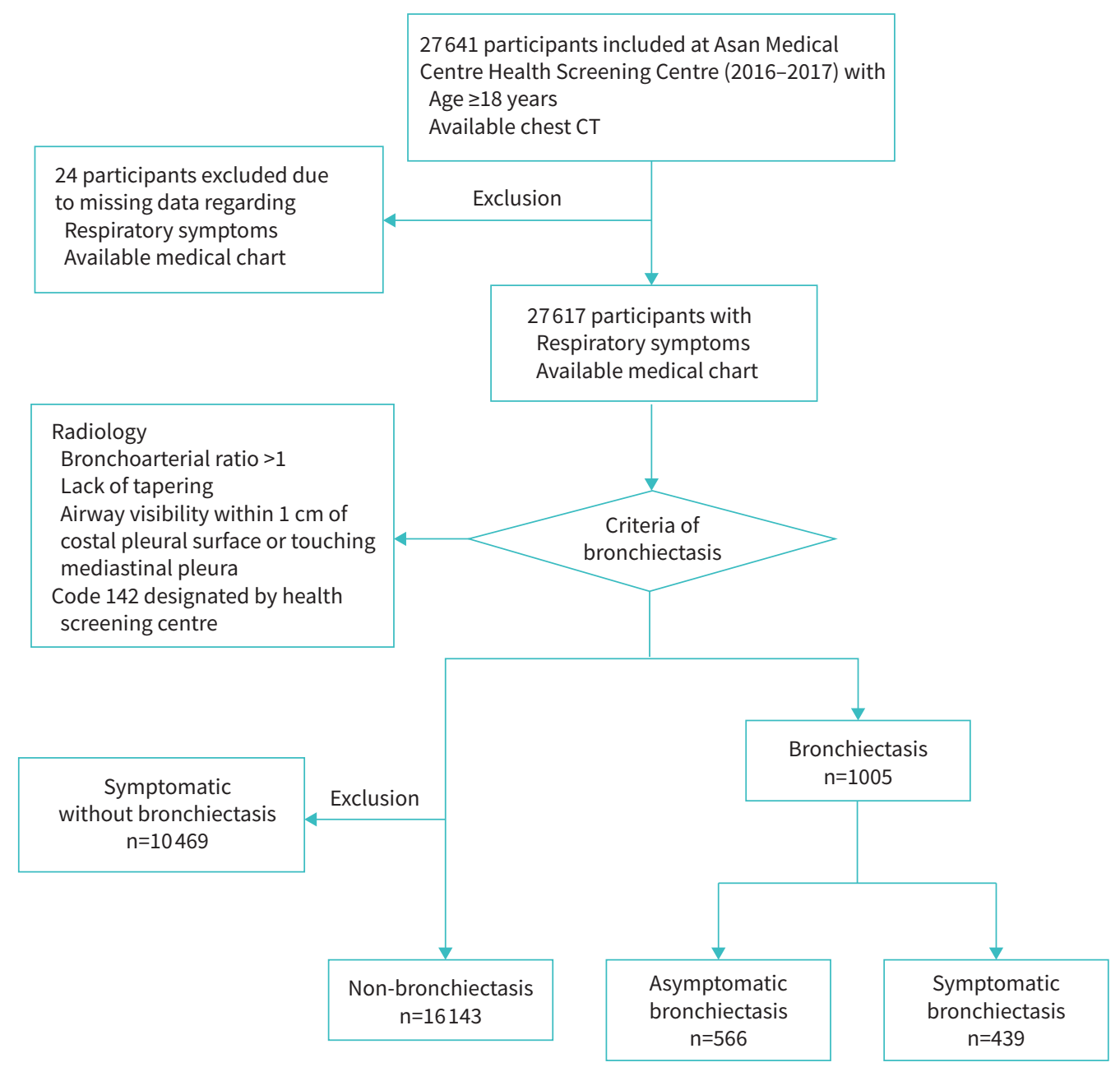

FIGURE 1 Flow chart of the study population. CT: computed tomography.

asymptomatic bronchiectasis group was 2050 out of 100000 , which was higher than that of the symptomatic group (1590 out of 100000). The prevalence of asymptomatic bronchiectasis, adjusted by age, sex and year, was 1235 out of 100000 , which was also higher than that in the symptomatic group (1094 out of 100000).

Factors related to asymptomatic bronchiectasis

Factors related to asymptomatic bronchiectasis were evaluated and compared with those in the non-bronchiectasis group (figure 4). Univariate analysis (presented as odds ratios; 95\% confidence intervals) revealed the associations for the following variables: female sex $(1.22 ; 1.03-1.45)$, age in years (1.06; 1.05-1.07), hypertension (1.17; 0.97-1.41), diabetes mellitus (1.25; 0.97-1.61), hyperlipidaemia (1.3; 1.08-1.58), arrhythmia (1.61; 1.08-2.42), CVD (1.69; 0.96-2.97), osteoporosis (1.58; 1.18-2.12), arthritis (1.3; 0.99-1.71), liver disease (1.34; 1.10-1.65), COPD (6.43; 3.84-10.79), tuberculosis (2.19; 1.64-2.94), smoking history (former smoker: 0.86; 0.71-1.03/current smoker: 0.61; 0.47-0.79), FEV (\% predicted) (0.99; 0.98-0.995) and haemoglobin levels (0.92; 0.87-0.98). Among these variables, multivariate analysis (presented as odds ratios; 95\% confidence intervals) showed that female sex (1.41; $1.18-1.70)$, older age (1.06; 1.05-1.07), comorbid liver disease (1.32; 1.07-1.63) and COPD (4.99; 2.88 8.64), history of tuberculosis (1.98; 1.46-2.68), and lower $\mathrm{FEV}_{1}$ (\% predicted) $(0.99 ; 0.98-0.998)$ were related to asymptomatic bronchiectasis.

Factors related to the presence of respiratory symptoms in bronchiectasis

Factors related to respiratory symptoms in bronchiectasis were evaluated, with comparison between the symptomatic bronchiectasis and asymptomatic bronchiectasis groups (supplementary figure S1). 
TABLE 1 Baseline characteristics of non-bronchiectasis, asymptomatic bronchiectasis and symptomatic bronchiectasis

\begin{tabular}{|c|c|c|c|c|c|c|c|}
\hline & \multirow[t]{2}{*}{ Non-bronchiectasis (A) } & \multirow{2}{*}{$\begin{array}{c}\text { Asymptomatic } \\
\text { bronchiectasis (B) }\end{array}$} & \multirow{2}{*}{$\begin{array}{c}\text { Symptomatic } \\
\text { bronchiectasis (C) }\end{array}$} & \multicolumn{4}{|c|}{$\mathrm{p}$-value } \\
\hline & & & & $\begin{array}{l}\text { A versus B } \\
\text { versus C }\end{array}$ & A versus $B$ & $B$ versus $C$ & A versus $\mathrm{C}$ \\
\hline Subjects $n$ & 16143 & 566 & 439 & & & & \\
\hline Female sex & 5555 (34.4) & $221(39.0)$ & $199(45.3)$ & $<0.001$ & 0.023 & 0.045 & $<0.001$ \\
\hline Age years & $58.19 \pm 8.58$ & $64.24 \pm 7.40$ & $62.64 \pm 7.71$ & $<0.001$ & $<0.001$ & 0.402 & $<0.001$ \\
\hline BMI $(n=16136 / 566 / 439)$ & & & & 0.580 & 0.605 & 0.249 & 0.410 \\
\hline Underweight $\left(\mathrm{BMI}<18.5 \mathrm{~kg} \cdot \mathrm{m}^{-2}\right.$ ) & $462(2.9)$ & $14(2.5)$ & $16(3.6)$ & & & & \\
\hline $\begin{array}{l}\text { Normal weight (BMI } \geqslant 18.5 \text { and } \\
<25 \mathrm{~kg} \cdot \mathrm{m}^{-2} \text { ) }\end{array}$ & $10926(67.7)$ & $394(69.6)$ & $286(65.1)$ & & & & \\
\hline $\begin{array}{l}\text { Overweight or obesity (BMI } \\
\geqslant 25 \mathrm{~kg} \cdot \mathrm{m}^{-2} \text { ) }\end{array}$ & $4748(29.4)$ & $158(27.9)$ & $137(31.2)$ & & & & \\
\hline Smoking history $(n=16090 / 563 / 437)$ & & & & $<0.001$ & $<0.001$ & 0.322 & 0.002 \\
\hline Never smoker & $7523(46.8)$ & $301(53.5)$ & $242(55.4)$ & & & & \\
\hline Former smoker & $5371(33.4)$ & $184(32.7)$ & $125(28.6)$ & & & & \\
\hline Current smoker & $3196(19.9)$ & $78(13.9)$ & $70(16.0)$ & & & & \\
\hline $\begin{array}{l}\text { PFT: } \text { FEV }_{1} \% \text { predicted }(n=15747 / \\
553 / 430)\end{array}$ & $92.06 \pm 11.18$ & $90.42 \pm 12.07$ & $85.63 \pm 14.69$ & $<0.001$ & 0.002 & $<0.001$ & 0.001 \\
\hline \multicolumn{8}{|l|}{ Laboratory test } \\
\hline $\begin{array}{l}\text { Haemoglobin } \mathrm{g} \cdot \mathrm{dL}^{-1}(\mathrm{n}=16140 / \\
566 / 439)\end{array}$ & $14.61 \pm 1.41$ & $14.44 \pm 1.42$ & $14.38 \pm 1.44$ & $<0.001$ & 0.006 & 0.537 & $<0.001$ \\
\hline $\mathrm{CRP} \mathrm{mg} \cdot \mathrm{dL}^{-1}(\mathrm{n}=14618 / 517 / 403)$ & $0.11 \pm 0.16$ & $0.11 \pm 0.15$ & $0.16 \pm 0.25$ & $<0.001$ & 0.363 & $<0.001$ & $<0.001$ \\
\hline
\end{tabular}

Continuous variables are presented as mean \pm SD; categorical variables are presented as number (\%). BMI: body mass index; CRP: C-reactive protein; $\mathrm{FEV}_{1}$ : forced expiratory volume in $1 \mathrm{~s}$; PFT: pulmonary function test.

\section{Factors related to symptomatic bronchiectasis}

Factors related to symptomatic bronchiectasis were evaluated, with comparison to the non-bronchiectasis group (supplementary figure S2).

\section{Discussion}

Our study reports the prevalence of bronchiectasis in South Korea based on CT findings, using the 20162017 database at a health screening centre of Asan Medical Centre. The data were further adjusted by age, sex and the corresponding year for the Korean general population. Furthermore, this is the first study to

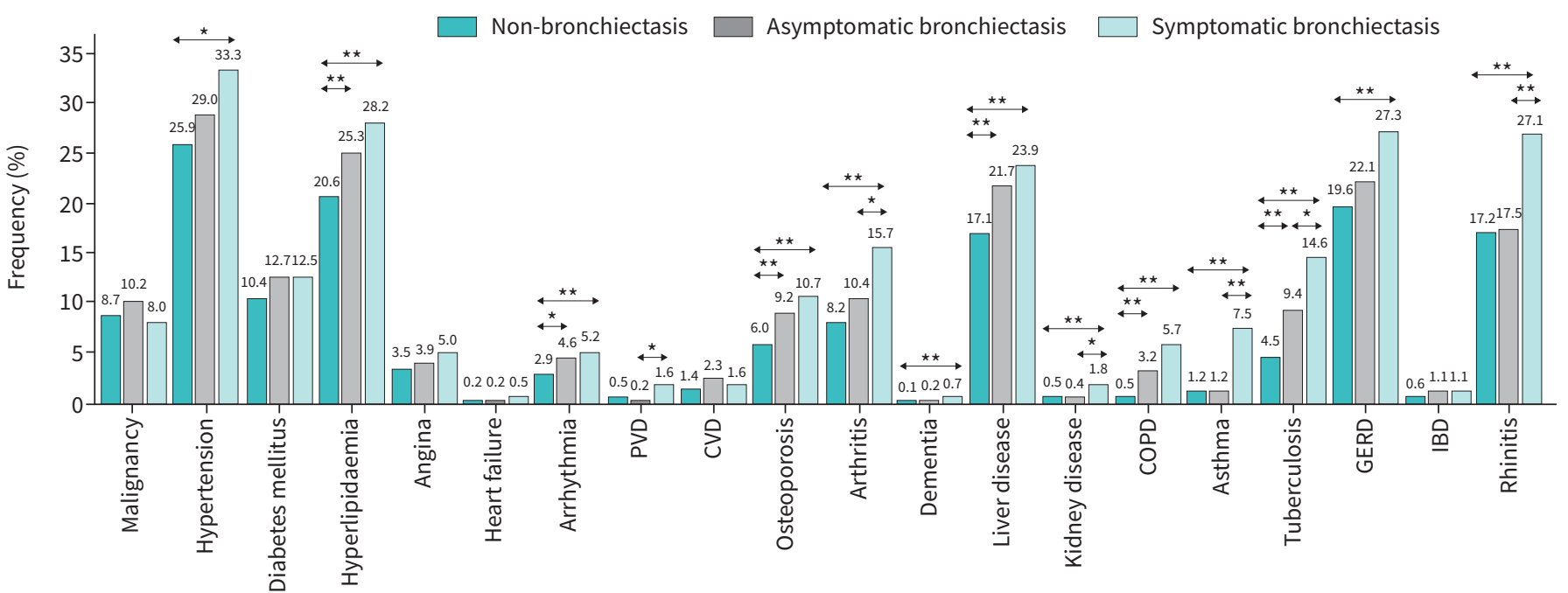

FIGURE 2 Frequency of comorbidities among non-bronchiectasis, asymptomatic bronchiectasis and symptomatic bronchiectasis. ${ }^{\star} p<0.05$, ${ }^{\star *} p<0.01$. PVD: peripheral vascular disease; CVD: cerebrovascular disease; GERD: gastro-oesophageal reflux disease; IBD: inflammatory bowel disease. 


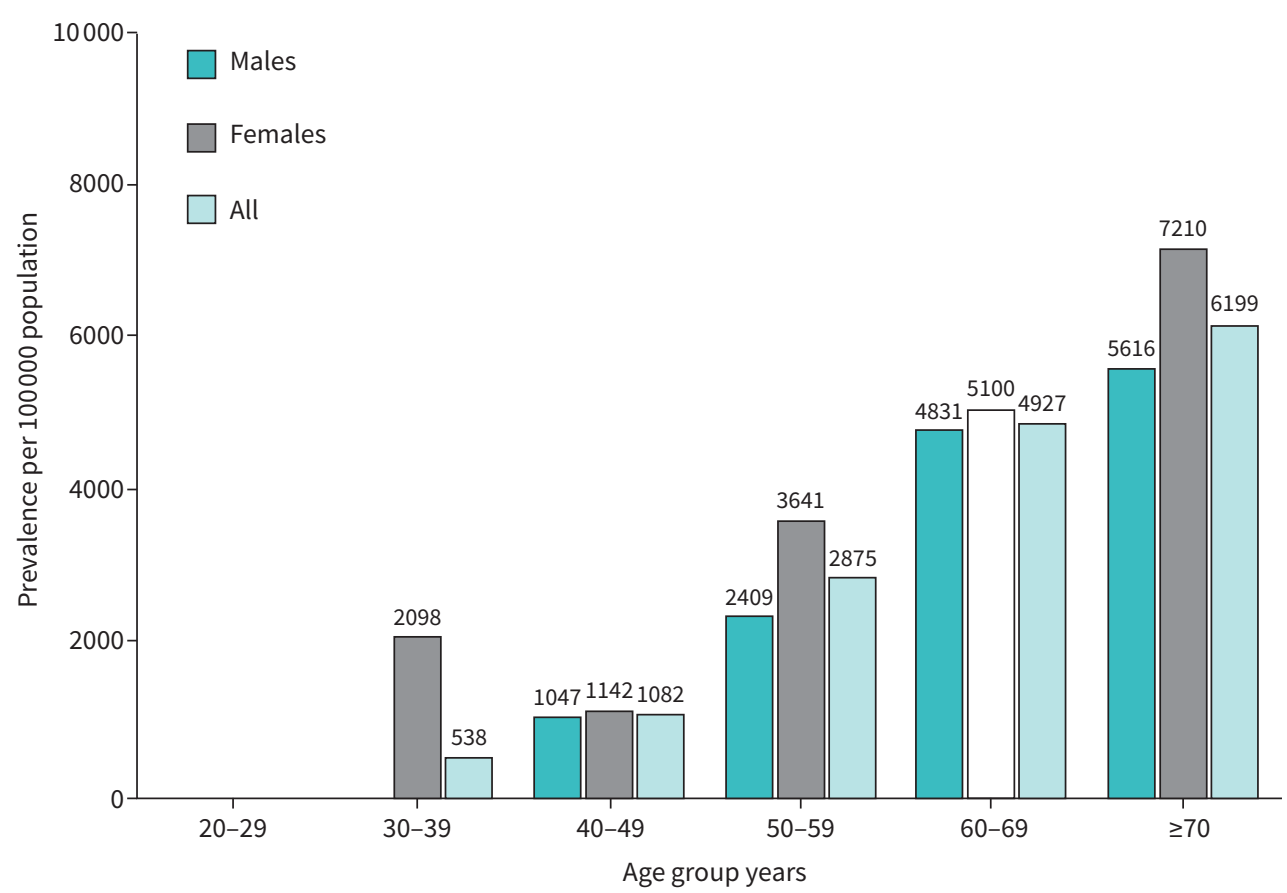

FIGURE 3 Prevalence of bronchiectasis based on age group and sex in South Korea during 2016-2017.

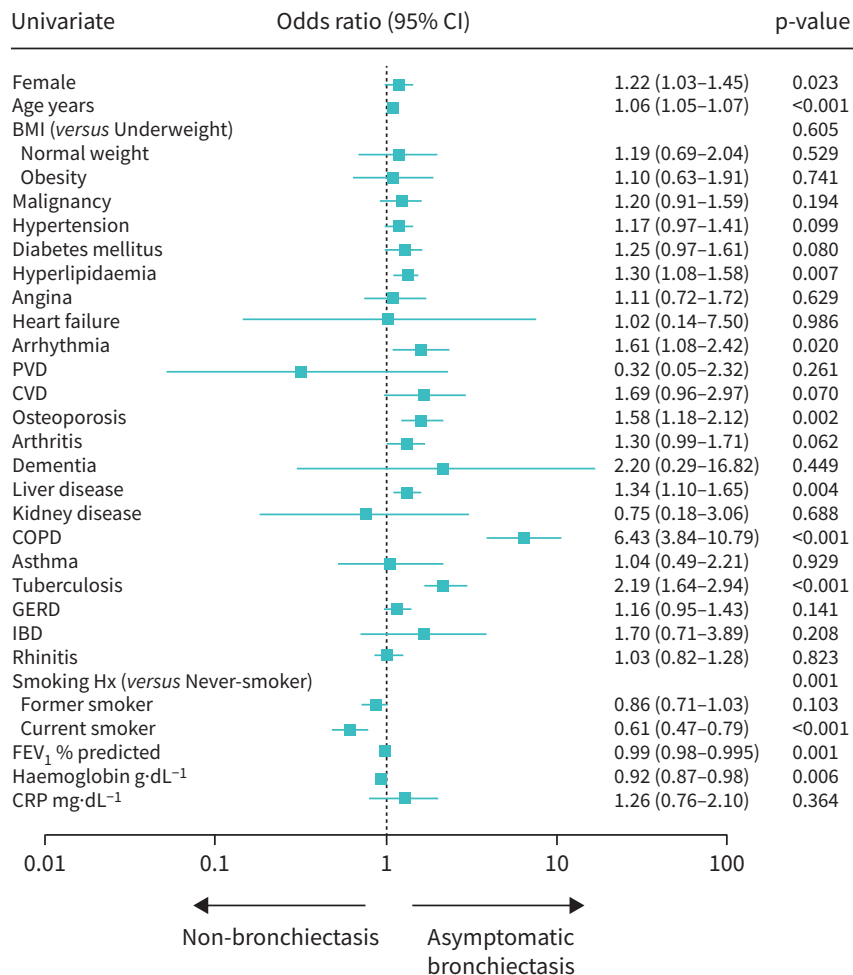

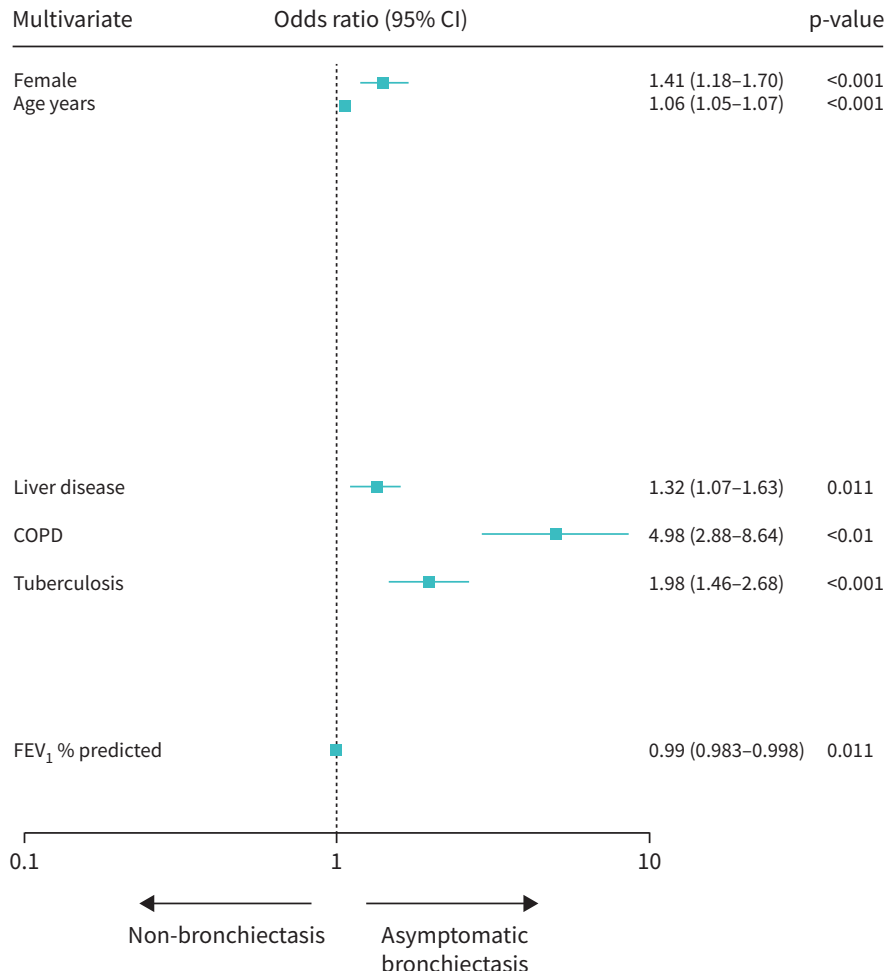

FIGURE 4 Factors related to asymptomatic bronchiectasis. BMI: body mass index; CRP: C-reactive protein; CVD: cerebrovascular disease; $\mathrm{FEV}_{1}$ : forced expiratory volume in $1 \mathrm{~s}$; GERD: gastro-oesophageal reflux disease; Hx: history; IBD: inflammatory bowel disease; PVD: peripheral vascular disease. 
evaluate the prevalence of asymptomatic bronchiectasis and its related factors, which were female sex, advanced age, comorbid liver disease and COPD, history of tuberculosis and low FEV . $_{\text {. }}$

In our study, the adjusted prevalence of bronchiectasis in South Korea was 2329 per 100000 , with female predominance and an increase with age. Some studies in South Korea have shown a lower prevalence of bronchiectasis than that reported in this study. A study by Cног et al. [20] reported the prevalence of bronchiectasis as 464 per 100000 population in South Korea. It was based on the 2012-2017 Health Insurance Review and Assessment Service, National Patient Sample (HIRA-NPS) database and the ICD-10 diagnostic code, which could underestimate the true prevalence of bronchiectasis. A study by YANG et al. [21] showed that the prevalence of bronchiectasis in subjects aged 40 or more years was $0.8 \%$, which was based on the 2007-2009 Korean National Health and Nutrition Examination Survey database. However, there was a possibility of recall bias and exclusion of patients with severe symptoms and inability to respond to the survey. Our study estimated the prevalence of bronchiectasis based on CT findings in all subjects, adjusted to the Korean general population. Thus, our study may provide a more accurate prevalence of bronchiectasis in South Korea.

Previous studies on the prevalence of bronchiectasis were mostly limited to western countries, producing a result lower than that reported in our study. The annual prevalence of bronchiectasis has been reported to be 486 (for males) to 566 (for females) per 100000 population in the UK from 2004 to 2013 [7], 163 per 100000 population in Italy from 2002 to 2015 [22], 95 per 100000 population in Germany from 2009 to 2017 [9] and 139 per 100000 population in the US in 2013 [23]. This inconsistency might be partially explained by ethnicity; Asians showed 2.5- and 3.9-fold higher prevalence of bronchiectasis than Caucasian and African-American populations among people aged $\geqslant 65$ years with one available CT scan, indicating a predisposition towards bronchiectasis in the Asian population [8]. The inconsistency might also be attributed to differences in the aetiology of bronchiectasis worldwide. In Asia, the prevalence of pulmonary infection and pulmonary tuberculosis were reported to be much higher than that in Western countries and accounted for the major aetiologies of bronchiectasis [24]. Further CT-based studies with international multicentric cohorts with a focus on genetic susceptibility and aetiologies of bronchiectasis are warranted to support a higher prevalence of bronchiectasis in Asia.

Another important finding of our study is that the adjusted prevalence of asymptomatic bronchiectasis was 1235 per 100000 , which was higher than that of the symptomatic group (1094 per 100000). Previous studies were mostly based on the ICD-10 diagnostic code, which might have excluded patients with asymptomatic bronchiectasis not requiring hospital visits [9, 20, 22, 23]. One study showed that asymptomatic bronchiectasis accounted for $46 \%$ of all bronchiectasis cases, but the total study population was small $(n=1409)$ [25]. Our study emphasises the high burden of asymptomatic bronchiectasis with stronger evidence because our large cohort from the health screening centre was likely more attentive to health issues during the asymptomatic period. Thus, our study indicates the need for increased awareness of asymptomatic bronchiectasis to prevent disease onset and slow progression. Further studies are warranted to evaluate the impact of asymptomatic bronchiectasis on the overall clinical course of bronchiectasis.

Interestingly, female sex was associated with asymptomatic bronchiectasis and also with the presence of respiratory symptoms in bronchiectasis. Female predominance in the prevalence of non-cystic bronchiectasis was previously reported, although there were conflicting results for sex predominance in the elderly population [7, 22, 23, 26]. This might be due to changes in the aetiologies of bronchiectasis, such as increased non-tuberculous mycobacterial infection and connective tissue disease, which are predominant in females [27-29]. Furthermore, some studies suggested that female anatomy and sex hormone factors might contribute to sex differences because of early bacterial colonisation and its conversion to the Pseudomonas phenotype in bronchiectasis, which might lead to development of respiratory symptoms [30, 31]. Thus, females are likely to have a relatively high risk of future bronchiectasis, as well as respiratory symptoms when bronchiectasis develops. More attention should be paid to this population, and further prospective cohort studies are warranted to evaluate the causal relationships with bronchiectasis.

Furthermore, liver disease was positively correlated with bronchiectasis, which might be mostly attributed to asymptomatic bronchiectasis. Among the aetiologies of liver disease, fatty liver disease was the most common cause in our study, comprising $75.2 \%$ (2078 out of 2764) in non-bronchiectasis, $71.5 \%$ (88 out of 123) in asymptomatic bronchiectasis and 71.5\% (88 out of 123) in symptomatic bronchiectasis groups. Non-alcoholic fatty liver disease (NAFLD) was previously reported to be highly prevalent in patients with COPD [32, 33] and positively associated with impaired lung function [34, 35], indicating a close relationship with airway disease. This might be attributed to systemic inflammation associated with 
NAFLD such as interleukin-8, tumour necrosis factor $\alpha$, leptin and oxidative stress [36-38], which might induce recurrent bronchial inflammation and the development of bronchiectasis. Thus, liver disease could be used to identify subjects with a high risk of developing asymptomatic bronchiectasis. Further prospective studies are warranted to evaluate the relationship between these factors and asymptomatic bronchiectasis.

Our study has some limitations. First, this study was based on voluntary participants at a tertiary centre, which may have caused a selection bias. Furthermore, this may have influenced the presence of certain comorbid conditions. However, this was a large population-based study adjusted to the standard population and may be representative of the general health screening population of South Korea. Second, true causality of the factors related to asymptomatic bronchiectasis could not be identified due to the retrospective and cross-sectional study design. Therefore, further prospective, longitudinal cohort studies are needed to confirm the true causal relationship of the potential risk factors for asymptomatic bronchiectasis. Third, we could not estimate the impact of asymptomatic bronchiectasis on clinical outcomes, such as mortality, hospitalisation, exacerbations and quality of life, due to the nature of data obtained from a health screening centre with a short study period. Further studies with adequate, longitudinal data on clinical outcomes are thus warranted to evaluate the association with asymptomatic bronchiectasis. Fourth, comorbidities might have been underestimated due to the retrospective nature of our study. Future studies with various aetiologies of bronchiectasis are needed to evaluate their effects on asymptomatic bronchiectasis. Fifth, in asymptomatic bronchiectasis, there was a limitation in discriminating dry bronchiectasis from early stage of symptomatic bronchiectasis due to the retrospective nature and absence of aetiological testing. Further studies are needed to define and evaluate the pre-clinical stage of symptomatic bronchiectasis.

\section{Conclusion}

Bronchiectasis might not be a rare disease in South Korea, where asymptomatic bronchiectasis appeared to be more common than symptomatic bronchiectasis. Female sex, older age, liver disease, COPD, history of tuberculosis and low $\mathrm{FEV}_{1}$ might be related to asymptomatic bronchiectasis. Therefore, the related factors should be further explored.

Acknowledgements: We would like to thank Seonok Kim (Department of Medical Statistics, Asan Medical Center, University of Ulsan College of Medicine) for her statistical advice. All authors meet the ICMJE authorship criteria.

Conflict of interest: None declared.

\section{References}

1 Chalmers JD, Aliberti S, Blasi F. Management of bronchiectasis in adults. Eur Respir J 2015; 45: 1446-1462.

2 Pasteur MC, Bilton D, Hill AT, British Thoracic Society Bronchiectasis (non-CF) Guideline Group. British Thoracic Society guideline for non-CF bronchiectasis. Thorax 2010; 65: Suppl. 1, i1-i58.

3 Dodd JD, Lavelle LP, Fabre A, et al. Imaging in cystic fibrosis and non-cystic fibrosis bronchiectasis. Semin Respir Crit Care Med 2015; 36: 194-206.

4 Hill AT, Sullivan AL, Chalmers JD, et al. British Thoracic Society Guideline for bronchiectasis in adults. Thorax 2019; 74: Suppl. 1, 1-69.

5 Goeminne PC, De Soyza A. Bronchiectasis: how to be an orphan with many parents? Eur Respir J 2016; 47: $10-13$.

6 Martínez García MA. Bronchiectasis: still an orphan disease? Arch Bronconeumol 2005; 41: 407-409.

7 Quint JK, Millett ER, Joshi M, et al. Changes in the incidence, prevalence and mortality of bronchiectasis in the UK from 2004 to 2013: a population-based cohort study. Eur Respir J 2016; 47: 186-193.

8 Seitz AE, Olivier KN, Adjemian J, et al. Trends in bronchiectasis among medicare beneficiaries in the United States, 2000 to 2007. Chest 2012; 142: 432-439.

9 Ringshausen FC, Rademacher J, Pink I, et al. Increasing bronchiectasis prevalence in Germany, 2009-2017: a population-based cohort study. Eur Respir J 2019; 54: 1900499.

10 Loebinger MR, Wells AU, Hansell DM, et al. Mortality in bronchiectasis: a long-term study assessing the factors influencing survival. Eur Respir J 2009; 34: 843-849.

11 de la Rosa D, Martínez-Garcia MA, Olveira C, et al. Annual direct medical costs of bronchiectasis treatment: impact of severity, exacerbations, chronic bronchial colonization and chronic obstructive pulmonary disease coexistence. Chron Respir Dis 2016; 13: 361-371.

12 Araújo D, Shteinberg M, Aliberti S, et al. Standardised classification of the aetiology of bronchiectasis using an objective algorithm. Eur Respir J 2017; 50: 1701289.

13 Barker AF. Bronchiectasis. N Engl J Med 2002; 346: 1383-1393. 
14 Aliberti S, Lonni S, Dore S, et al. Clinical phenotypes in adult patients with bronchiectasis. Eur Respir J 2016; 47: 1113-1122.

15 Chalmers JD, Chotirmall SH. Bronchiectasis: new therapies and new perspectives. Lancet Respir Med 2018; 6: 715-726.

16 Park GM, Han S, Kim SH, et al. Model for assessing cardiovascular risk in a Korean population. Circ Cardiovasc Qual Outcomes 2014; 7: 944-951.

17 Novosad S, Khan S, Wolfe B, et al. Role of obesity in asthma control, the obesity-asthma phenotype. J Allergy (Cairo) 2013; 2013: 538642

18 Laszlo G. Standardisation of lung function testing: helpful guidance from the ATS/ERS Task Force. Thorax 2006; 61: 744-746.

19 Statistics Korea. Population by age and sex 2016-2017. https://kosis.kr/statHtml/statHtml.do? orgld=101\&tblld=DT_1IN1503\&conn_path=I2 Date last updated: 24 November 2020. Date last accessed: 8 February 2021.

20 Choi H, Yang B, Nam H, et al. Population-based prevalence of bronchiectasis and associated comorbidities in South Korea. Eur Respir J 2019; 54: 1900194.

21 Yang B, Choi $\mathrm{H}$, Lim JH, et al. The disease burden of bronchiectasis in comparison with chronic obstructive pulmonary disease: a national database study in Korea. Ann Transl Med 2019; 7: 770.

22 Aliberti S, Sotgiu G, Lapi F, et al. Prevalence and incidence of bronchiectasis in Italy. BMC Pulm Med 2020; $20: 15$.

23 Weycker D, Hansen GL, Seifer FD. Prevalence and incidence of noncystic fibrosis bronchiectasis among US adults in 2013. Chron Respir Dis 2017; 14: 377-384.

24 Gao YH, Guan WJ, Liu SX, et al. Aetiology of bronchiectasis in adults: a systematic literature review. Respirology 2016; 21: 1376-1383.

25 Kwak HJ, Moon JY, Choi YW, et al. High prevalence of bronchiectasis in adults: analysis of CT findings in a health screening program. Tohoku J Exp Med 2010; 222: 237-242.

26 Monteagudo M, Rodriguez-Blanco T, Barrecheguren M, et al. Prevalence and incidence of bronchiectasis in Catalonia, Spain: a population-based study. Respir Med 2016; 121: 26-31. Whitacre CC. Sex differences in autoimmune disease. Nat Immunol 2001; 2: 777-780.

28 Kim RD, Greenberg DE, Ehrmantraut ME, et al. Pulmonary nontuberculous mycobacterial disease: prospective study of a distinct preexisting syndrome. Am J Respir Crit Care Med 2008; 178: 1066-1074.

29 Chan ED, Iseman MD. Underlying host risk factors for nontuberculous mycobacterial lung disease. Semin Respir Crit Care Med 2013; 34: 110-123.

30 Raghavan D, Jain R. Increasing awareness of sex differences in airway diseases. Respirology 2016; 21: 449-459.

31 Vidaillac C, Yong VFL, Jaggi TK, et al. Gender differences in bronchiectasis: a real issue? Breathe (Sheff) 2018; 14: $108-121$

32 Moon SW, Kim SY, Jung JY, et al. Relationship between obstructive lung disease and non-alcoholic fatty liver disease in the Korean population: Korea National Health and Nutrition Examination Survey, 2007-2010. Int J Chron Obstruct Pulmon Dis 2018; 13: 2603-2611.

33 Viglino D, Jullian-Desayes I, Minoves $\mathrm{M}$, et al. Nonalcoholic fatty liver disease in chronic obstructive pulmonary disease. Eur Respir J 2017; 49: 1601923.

34 Mantovani A, Lonardo A, Vinco G, et al. Association between non-alcoholic fatty liver disease and decreased lung function in adults: a systematic review and meta-analysis. Diabetes Metab 2019; 45: 536-544.

35 Song JU, Jang Y, Lim SY, et al. Decreased lung function is associated with risk of developing non-alcoholic fatty liver disease: a longitudinal cohort study. PLoS One 2019; 14: e0208736.

36 Polyzos SA, Mantzoros CS. Nonalcoholic fatty future disease. Metabolism 2016; 65: 1007-1016.

37 Kugelmas M, Hill DB, Vivian B, et al. Cytokines and NASH: a pilot study of the effects of lifestyle modification and vitamin E. Hepatology 2003; 38: 413-419.

38 Sumida Y, Niki E, Naito Y, et al. Involvement of free radicals and oxidative stress in NAFLD/NASH. Free Radic Res 2013; 47: 869-880. 